\title{
RESEARCH
}

\section{Efficacy and safety of unfractionated heparin versus enoxaparin: a pooled analysis of ASSENT- 3 and -3 PLUS data}

\author{
Paul W. Armstrong, Wei-Ching Chang, Lars Wallentin, Patrick Goldstein, Christopher B. Granger, \\ Kris Bogaerts, Thierry Danays, Frans Van de Werf, for the ASSENT-3 and ASSENT-3 PLUS Investigators
}

$\infty \quad$ See related article page I43I

\section{ABstraCT}

Background: The optimal antithrombotic therapy to accompany tenecteplase in cases of acute ST-segment elevation myocardial infarction (STEMI) remains unclear. We undertook a prespecified pooled analysis of data from the ASSENT3 and ASSENT-3 PLUS trials.

Methods: We created a combined database of the 2040 and 818 patients who received enoxaparin in ASSENT- 3 and ASSENT-3 PLUS, respectively, and compared them with the 2038 and 821 patients who received unfractionated heparin.

Results: The primary efficacy end point, a composite of 30day mortality, reinfarction or refractory ischemia, was $16.0 \%$ with enoxaparin versus $\mathbf{1 2 . 2} \%$ with unfractionated heparin ( $p$ $<0.001$ ); the efficacy plus safety (intracranial hemorrhage $[\mathrm{ICH}]$ or major systemic bleeding) end point, $18.0 \%$ versus $15.0 \%(p=0.003)$. The 1049 patients urgently revascularized had greater benefit from enoxaparin $(15.4 \%$ v. $10.1 \%, p=$ $0.013)$, yet the excess in major systemic bleeding evident with enoxaparin (3.3\% v. $2.4 \%, p=0.01$ ) was largely confined to the 3492 patients without or before revascularization. Although $\mathrm{ICH}$ rates in the groups were similar (1.3\% v. $0.9 \%, p=$ 0.26 ), an excess of $\mathrm{ICH}$ occurred among those administered enoxaparin during the ASSENT-3 PLUS trial (6.7\% v. $0.8 \%, p=$ $0.013)$, especially among women over 75 years of age.

Interpretation: These data demonstrated the benefit of enoxaparin used in conjunction with tenecteplase, but raised caution about its prehospital use to treat STEMI in elderly women.

CMAJ 2006;174(10):I42I-6

$\mathrm{T}$ he best antithrombotic therapy to accompany fibrinolysis for acute ST-segment elevation myocardial infarction (STEMI) remains uncertain. In ASSENT-3, the Assessment of the Safety and Efficacy of a New Thrombolytic Agent trial, we showed that the combination of enoxaparin and tenecteplase was more effective than unfractionated heparin plus tenecteplase in reducing ischemic complications in patients treated within 6 hours of onset of symptoms of STEMI. ${ }^{1}$ Although patients treated with enoxaparin experienced a modest excess in the incidence of major systemic bleeding, the enoxaparin-tenecteplase combination was administered continuously until either hospital discharge or revascularization (maximum 7 days), whereas unfractionated heparin plus tenecteplase was terminated after 48 hours. Because the incidence of intracranial hemorrhage (0.9\%) was the same with either treatment strategy and was commensurate with rates found in other studies of fibrinolytic agents, we asserted that enoxaparin was an attractive alternative antithrombotic and deserved further study.

In a planned extension of ASSENT-3 involving patients with the same clinical characteristics in the prehospital setting (ASSENT-3 PLUS), tenecteplase administration was accompanied by either unfractionated heparin or enoxaparin. We confirmed that the enoxaparin combination reduced reinfarction and recurrent ischemia more than the unfractionated heparin combination, with an excess in major systemic bleeding in keeping with that seen in ASSENT-3. We also observed an unexpected increase in intracranial hemorrhage among the patients in the enoxaparin group who were older than 75 years. ${ }^{2}$

We now report on a pooled analysis (with a prespecified strategy) of data from ASSENT- 3 and ASSENT-3 PLUS to provide an overall efficacy and safety profile of these 2 antithrombotic strategies (i.e., enoxaparin and unfractionated heparin) used in conjunction with tenecteplase to treat STEMI.

\section{Methods}

Our findings from ASSENT- 3 and ASSENT- 3 PLUS have been previously reported. ${ }^{1,2}$ In brief, patients with STEMI were randomly assigned in ASSENT- 3 to receive a single intravenous (IV) bolus of tenecteplase in combination with enoxaparin, abciximab plus low-dose unfractionated heparin, or a typical dose of unfractionated heparin. In ASSENT-3 PLUS, patients with STEMI were randomly assigned to receive a single IV bolus of tenecteplase with either enoxaparin or unfractionated heparin in the prehospital setting. For the current pooled analysis, we included ASSENT- 3 data only from the groups receiving enoxaparin $(n=2040)$ or unfractionated heparin $(n=2038)$. ASA ( $150-325 \mathrm{mg}$ ) was given to all patients, who continued taking it daily at a similar dose. A weight-adjusted dose of tenecteplase (30 mg if the patient weighed $<60 \mathrm{~kg}, 35 \mathrm{mg}$ if $60-69 \mathrm{~kg}$, $40 \mathrm{mg}$ if $70-79 \mathrm{~kg}, 45 \mathrm{mg}$ if $80-89 \mathrm{~kg}$ and $50 \mathrm{mg}$ if $\geq 90 \mathrm{~kg}$ ) was administered over a 5 -second period. Patients assigned to 
the unfractionated heparin group received a 6o-unit/kg bolus (maximum 4000 units) and an initial infusion of $\mathrm{I} 2$ units $/ \mathrm{kg}$ per hour (maximum Iooo units/h), which was adjusted to maintain an activated partial thromboplastin time (aPTT) of 50-70 seconds for 48 hours. Those assigned to the enoxaparin group received an IV bolus of $30 \mathrm{mg}$ followed by a subcutaneous dose of I $\mathrm{mg} / \mathrm{kg}$; the subcutaneous dose was repeated every 12 hours until hospital discharge or revascularization (maximum 7 days); the first 2 subcutaneous doses had an upper limit of $100 \mathrm{mg}$. Patients involved in the ASSENT-3 PLUS trial were similarly evaluated, randomly assigned and treated in the prehospital setting, in accordance with local resources.

The 2 trials had 2 primary end points, both composites: efficacy (30-day mortality, in-hospital reinfarction or refractory ischemia); and efficacy plus safety (the aforementioned efficacy end points, plus in-hospital intracranial hemorrhage or other major bleeding). An independent Stroke Evaluation Panel unaware of group assignments reviewed all cases of suspected stroke; the investigators ascertained the other end points. ${ }^{1}$

We specified a detailed statistical analysis plan beforehand for a combined intention-to-treat analysis of the ASSENT-3 PLUS treatment groups and the correspondinga two ASSENT3 groups. This included pooled estimates of both primary efficacy and safety end points and their single components, in addition to between-group comparisons, to be presented as odds ratios (ORs) with confidence limits. We specified and used backward stepwise logistic regression to estimate adjusted ORs and 95\% confidence intervals (CIs) for these end points, adjusted not only for treatment-group assignment but also for baseline patient characteristics: age, sex, body height and weight; presence of diabetes or hypertension; heart rate and systolic blood pressure; Killip class; and time interval until treatment. ${ }^{3}$ For purposes of performing logistic regression analyses, another category was created for the $2 \mathrm{r} \%$ of ASSENT-3 PLUS participants whose Killip class data were missing. Kaplan-Meier survival analyses with log-rank tests were performed to test the differences between the treatment arms. We also undertook post-hoc subgroup analyses to explore the possible effect of urgent and nonurgent revascularization on the relations between the treatment agents and 2 in-hospital outcomes (reinfarction and major systemic bleeding) by data censoring at the time of revascularization. All statistical tests were 2 -sided, with the level of significance set at $5 \%$.

\section{Results}

Baseline characteristics (Table I; note that a more detailed version is available online as Appendix I at www.cmaj.ca/cgi /content/full/I74/Io/I42I/DCI) and concomitant medication use (Table 2) are shown for participants in the ASSENT-3 and ASSENT- 3 PLUS trials. Patients in ASSENT- 3 PLUS were slightly older, with a greater proportion older than 75 years, especially among the women. There were also more patients in ASSENT-3 PLUS with anterior myocardial infarctions (MIs) and a greater use of statins and thienopyridine therapy.

Table 3 shows the frequency of in-hospital events and use of invasive cardiac procedures. The frequency of invasive cardiac procedures, and more particularly of urgent and nonurgent
PCI, were notably higher among ASSENT-3 PLUS participants.

Fig. I presents a pooled analysis of the primary efficacy and the composite of primary efficacy plus safety end points; selected individual components of these are shown according to antithrombotic treatment assignment. A consistent reduction in both reinfarction and recurrent ischemia was evident in the enoxaparin group, with no significant effect on the rate of death; this constitutes the principal beneficial effect of this antithrombotic on the primary efficacy end point. Of note, I3 of the 97 patients who died during ASSENT-3 PLUS expired before hospital admission; these deaths were evenly balanced between the treatment groups (i.e., 6 patients who died received enoxaparin and 7 , unfractionated heparin). If these deaths are excluded, the mortality was $5.2 \%$ in the unfractionated heparin group and $6.8 \%$ in the enoxaparin group. The $\mathrm{I} \%$ absolute increase in major systemic bleeding and in intracranial hemorrhage evident with enoxaparin in ASSENT-3 PLUS lessen, but do not cancel, its favourable effect on the primary efficacy and safety end point. These findings were maintained after baseline adjustments: OR I.40 (95\% CI I.I9-I.64) for the primary efficacy end point and I.25 (95\% CI

Table 1: Baseline characteristics of patients in the ASSENT-3 PLUS and ASSENT-3 trials

\begin{tabular}{|c|c|c|c|c|}
\hline \multirow[b]{3}{*}{ Characteristic } & \multicolumn{4}{|c|}{$\%$ of treatment group* } \\
\hline & \multicolumn{2}{|c|}{ ASSENT-3 PLUS } & \multicolumn{2}{|c|}{ ASSENT-3 } \\
\hline & $\begin{array}{l}\text { Enox. } \\
n=818\end{array}$ & $\begin{array}{c}\text { UFH } \\
n=821\end{array}$ & $\begin{array}{c}\text { Enox. } \\
n=2040\end{array}$ & $\begin{array}{c}\text { UFH } \\
n=2038\end{array}$ \\
\hline Age, yr, mean (SD) & $62(13)$ & $62(13)$ & $61(12)$ & $61(13)$ \\
\hline$\%$ of patients $>75 \mathrm{yr}$ & 18 & 16 & 13 & 13 \\
\hline Women & 24 & 22 & 23 & 23 \\
\hline$\%$ of women $>75 \mathrm{yr}$ & 38 & 30 & 24 & 27 \\
\hline Hypertension & 35 & 36 & 41 & 41 \\
\hline Diabetes & 14 & 16 & 19 & 18 \\
\hline Previous MI & 15 & 14 & 14 & 14 \\
\hline Prior CABG & 2.6 & 1.6 & 3.6 & 2.6 \\
\hline Prior $\mathrm{PCl}$ & 7.4 & 6.4 & 6.2 & 6.4 \\
\hline Current smoker & 42 & 45 & 44 & 47 \\
\hline \multicolumn{5}{|l|}{ Infarct location } \\
\hline Anterior & 42 & 43 & 39 & 38 \\
\hline Inferior & 54 & 54 & 56 & 57 \\
\hline Other & 4.0 & 3.2 & 4.6 & 5.0 \\
\hline $\begin{array}{l}\text { Heart rate, beats } / \mathrm{min} \text {, } \\
\text { mean (SD) }\end{array}$ & $74(19)$ & 74 (19) & $75(17)$ & $74(17)$ \\
\hline $\begin{array}{l}\text { Systolic BP, mm Hg, } \\
\text { mean (SD) }\end{array}$ & $134(25)$ & $132(25)$ & $134(22)$ & $133(23)$ \\
\hline Killip class I† & 92 & 92 & 89 & 88 \\
\hline
\end{tabular}

Note: A more detailed version of this table is available online (at www.cmaj.ca /cgi/content/full/174/10/1xxx/DC1) as Appendix 1. ASSENT = Assessment of the Safety and Efficacy of a New Thrombolytic Agent, Enox. = enoxaparin, UFH = unfractionated heparin, $\mathrm{SD}=$ standard deviation, $\mathrm{MI}=$ myocardial infarction, CABG = coronary artery bypass graft, $\mathrm{PCl}=$ percutaneous coronary intervention, $\mathrm{BP}=$ blood pressure

*Unless indicated otherwise.

†Killip class was unrecorded in the records of $21 \%$ of ASSENT-3 PLUS patients. 
I.07-I.45) for the primary efficacy plus safety end point. Similar efficacy patterns were evident in subsets of patient data categorized by age, sex and presence of diabetes.

Data showing the beneficial effect of enoxaparin on in-

Table 2: Concomitant medication of patients, no. (\%), in the ASSENT-3 PLUS and ASSENT-3 trials.

\begin{tabular}{|c|c|c|c|c|}
\hline \multirow[b]{3}{*}{ Medication } & \multicolumn{4}{|c|}{ No. of patients (\%) } \\
\hline & \multicolumn{2}{|c|}{ ASSENT-3 PLUS } & \multicolumn{2}{|c|}{ ASSENT-3 } \\
\hline & $\begin{array}{c}\text { Enoxaparin } \\
n \simeq 815^{*}\end{array}$ & $\begin{array}{l}\text { UF heparin } \\
n \simeq 818 \dagger\end{array}$ & $\begin{array}{c}\text { Enoxaparin } \\
n \simeq 2034 \ddagger\end{array}$ & $\begin{array}{l}\text { UF heparin } \\
n \simeq 2030 \S\end{array}$ \\
\hline Nitrates, intravenous & $495(61)$ & $507(62)$ & $1490(73)$ & $1489(73)$ \\
\hline$\beta$-Blocker & $734(90)$ & $725(89)$ & $1707(84)$ & $1696(83)$ \\
\hline ACE or other inhibitor & $509(62)$ & $539(66)$ & $1300(64)$ & $1303(64)$ \\
\hline Statin & $604(74)$ & $574(70)$ & $1062(52)$ & $1024(51)$ \\
\hline ASA & $784(96)$ & $792(97)$ & $1969(97)$ & $1974(97)$ \\
\hline Ticlopidine or clopidogrel & $444(55)$ & $455(56)$ & $605(30)$ & $649(32)$ \\
\hline Oral anticoagulant & 37 (4.5) & $34 \quad(4.2)$ & $87 \quad(4.3)$ & $105(5.2)$ \\
\hline GP-IIb/-IIla inhibitor & $147(18)$ & $170(21)$ & $262(13)$ & $312(15)$ \\
\hline LMW heparinף & $268(33)$ & $389(47)$ & $325(16)$ & $584(29)$ \\
\hline Thrombolytic agentף & $20 \quad(2.4)$ & $17 \quad(2.1)$ & $40 \quad(2.0)$ & $58(2.9)$ \\
\hline
\end{tabular}

Note: ASSENT = Assessment of the Safety and Efficacy of a New Thrombolytic Agent, UF = unfractionated, $\mathrm{ACE}=$ angiotensin-converting enzyme, $\mathrm{GP}=$ glycoprotein, $\mathrm{LMW}=$ low molecular weight.

*Range of $n$ was 813-817.

†Range of $n$ was $816-820$

$\ddagger$ Range of $n$ was 2027-2040.

§Range of $n$ was 2023-2036.

१Other than the study drug.

Table 3: In-hospital cardiac events and invasive procedures in the ASSENT-3 PLUS and ASSENT-3 trials

\begin{tabular}{|c|c|c|c|c|}
\hline \multirow[b]{3}{*}{ Status } & \multicolumn{4}{|c|}{ No. (\%) } \\
\hline & \multicolumn{2}{|c|}{ ASSENT-3 PLUS } & \multicolumn{2}{|c|}{ ASSENT-3 } \\
\hline & Enoxaparin & UF heparin & Enoxaparin & UF heparin \\
\hline Cardiac event & $n=818$ & $n=821$ & $n=2040$ & $n=2038$ \\
\hline Sustained hypotension & $16 \quad(2.0)$ & $25 \quad(3.0)$ & $42 \quad(2.1)$ & $55 \quad(2.7)$ \\
\hline $\begin{array}{l}\text { Pulmonary edema or } \\
\text { cardiogenic shock }\end{array}$ & $43 \quad(5.3)$ & $52 \quad(6.3)$ & $106 \quad(5.2)$ & $115(5.6)$ \\
\hline Major arrhythmia & $71 \quad(8.7)$ & $81 \quad(9.9)$ & $173 \quad(8.5)$ & $212(10.4)$ \\
\hline Stroke & $24 \quad(2.9)$ & 11 (1.3) & $33(1.6)$ & $31 \quad(1.5)$ \\
\hline Procedure & $n \simeq 815^{*}$ & $n=813$ & $n \simeq 2037^{*}$ & $n \simeq 2035^{*}$ \\
\hline Intra-aortic balloon pump & 13 & 19 (2.3) & $53(2.6)$ & $56 \quad(2.8)$ \\
\hline CABG, urgent & $8 \quad(1.0)$ & $15 \quad(1.8)$ & $34 \quad(1.7)$ & 34 (1.7) \\
\hline CABG, elective & $19 \quad(2.3)$ & $21 \quad(2.6)$ & $48 \quad(2.4)$ & 71 (3.5) \\
\hline $\mathrm{PCl}$, urgent & $204(25.0)$ & $239(29.4)$ & 242 (11.9) & $292(14.3)$ \\
\hline $\mathrm{PCl}$, elective & $190(23.3)$ & $183(22.5)$ & $355(17.4)$ & $335(16.5)$ \\
\hline All procedures & $413(50.7)$ & $446(54.9)$ & $661(32.4)$ & $718(35.3)$ \\
\hline
\end{tabular}

Note: ASSENT = Assessment of the Safety and Efficacy of a New Thrombolytic Agent, UF = unfractionated, $\mathrm{CABG}$ = coronary artery bypass graft, $\mathrm{PCI}$ = percutaneous coronary

intervention.

${ }^{*} \pm 1$ patient, according to the data available. hospital reinfarction up until I4 days after group assignment, according to whether patients underwent in-hospital revascularization, is available in online Appendix 2 (www.cmaj.ca/cgi /content/full/174/Io/I42I/DCI). In this analysis, the no-revascularization cohort was defined for all patients by censoring on the right at the time of revascularization. Rates of reinfarction according to KaplanMeier estimates were reduced more among patients undergoing urgent revascularization $(9.2 \%$ enoxaparin v. $15.4 \%$ unfractionated heparin, $p=0.003$ ) than among those with nonurgent (I.5\% enoxaparin versus $2.7 \%$ unfractionated heparin, $p=0.15$ ) or without or before revascularization (I. $3 \%$ enoxaparin v. I. $7 \%$ unfractionated heparin, $p=0.19$ ). The median time to urgent revascularization was 0 days (interquartile range [IQR] 0-1 days); for nonurgent revascularization, 5 (IQR 2-9) days. Paradoxically, although excess bleeding occurred in both groups undergoing revascularization, the excess rate of major bleeding associated with enoxaparin was greater among patients either not undergoing or before revascularization $(2.0 \%$ enoxaparin v. $0.9 \%$ unfractionated heparin, $p=0.001$; see online Appendix 3, www.cmaj.ca/cgi/content/full/I74/Io/I42I/DCI). These findings remained unchanged after adjusting for baseline factors (age, sex, diabetes, MI location, heart rate and systolic blood pressure) and use of glycoprotein IIb/IIIa inhibitors, thienopyridines, thrombolytics or heparin.

Fig. 2 shows the predictors of intracranial hemorrhage. Age of 65 years or more, with a progressive incremental risk above 75 years, and systolic blood pressure of $150 \mathrm{~mm} \mathrm{Hg}$ or higher were significant predictors of intracranial hemorrhage. A tendency toward increased risk was also evident among those treated between 2 and 4 hours after the onset of symptoms of MI. Among women older than 75 years, intracranial hemorrhage was much more common with enoxaparin (10/183 such patients, or $5.5 \%$ ) than with unfractionated heparin (I/I85 or $0.5 \% ; p=0.005$ ). Moreover, the interaction between age, sex and enoxaparin treatment for the occurrence of intracranial hemorrhage was significant ( $p$ $=0.00 \mathrm{I}$ ). When rates of intracranial hemorrhage were examined by trial and according to treatment (Fig. 3), a significant excess occurred among patients assigned to the enoxaparin group in ASSENT3 PLUS, but not in ASSENT-3 ( $p=0.005$ for interaction between age, sex, treatment assignment, and trial in the pooled analysis).

\section{Interpretation}

This large, pooled analysis comparing 2 antithrombotics used in conjunction with tenecteplase to treat STEMI in over 5700 patients in 2 separate studies demonstrated the substantial therapeutic effect of enoxaparin in the prevention of recurrent MIs and 
refractory ischemia in hospital. The new data we have reported here supported an increased benefit of enoxaparin among patients with STEMI who were undergoing revascularization; unexpectedly, most of the bleeding risk was among patients not undergoing revascularization. We also found an important 3-way interaction between age, female sex and enoxaparin that contributed to the risk of intracranial hemorrhage.

An increase in intracranial hemorrhage among patients with STEMI who were treated prehospital with tenecteplase and enoxaparin seems surprising, given that it was not evident in the larger population of patients treated in hospital. Women older than 75 years in the enoxaparin group were at an especially high risk of intracranial hemorrhage. Since patients who summon an ambulance for transport to an emergency department are at higher risk than those who arrive by other means, these observations may relate to differences in these populations. ${ }^{4,5}$ Further evidence suggesting that prehospital patients are at greater risk was our finding that 13 of the 97 deaths dur- ing ASSENT-3 PLUS were prehospital; such data would have been excluded from the ASSENT-3 study. Although this rate of prehospital death was higher than rates reported from recent trials involving patients with STEMI who were transferred from community to tertiary care centres, ${ }^{6,7}$ prescreening of severely ill patients may, unlike ASSENT-3 PLUS, have resulted in patient exclusion from those studies. Moreover, patients involved in ASSENT-3 PLUS were far more likely to receive thienopyridine therapy and glycoprotein IIb/IIIa inhibitors. Since $39 \%$ of patients assigned to receive unfractionated heparin in ASSENT3 PLUS did not receive a prehospital infusion after the bolus, and nearly half of them had subtherapeutic initial aPTTs, the differences between the 2 antithrombotic strategies may have been further amplified. Despite a reduction in unfractionated heparin in both the IV bolus and infusion rates undertaken in ASSENT-3 (in accordance with the American College of Cardiology-American Heart Association guidelines for treatment of STEMI), no reduction in intracranial hemorrhage was evident

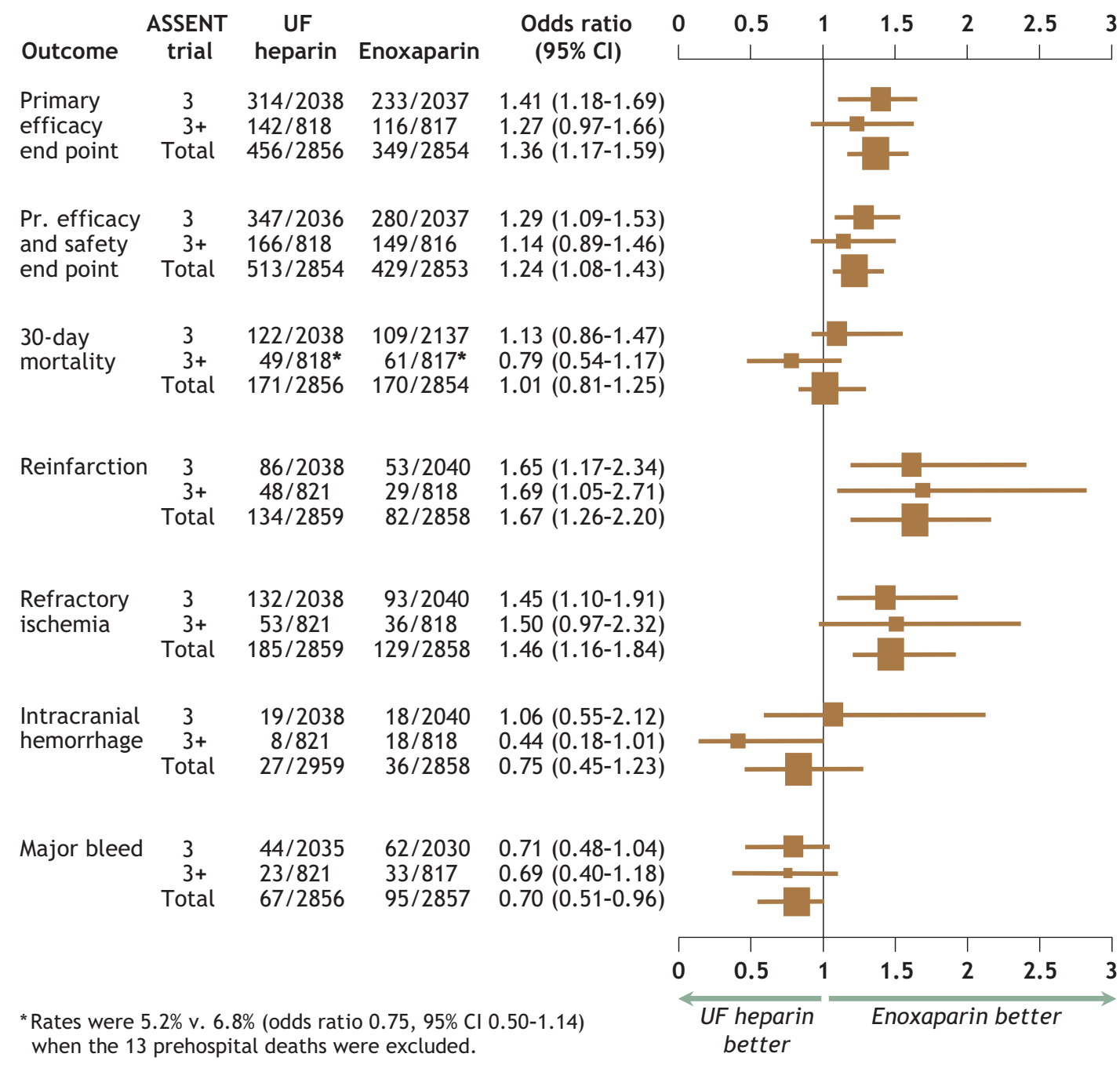

Fig. 1: Odds ratios and $95 \%$ confidence intervals $(\mathrm{Cl})$ for individual and composite efficacy and safety end points for ASSENT-3 (indicated as 3), ASSENT-3 PLUS ( $3+$ ) and pooled (Total) data. Data are plotted as odds ratios (the dimensions of the boxes represent relative population sizes) with $95 \%$ confidence intervals (bars). ASSENT $=$ Assessment of the Safety and Efficacy of a New Thrombolytic Agent, UF = unfractionated, Pr. = primary. 


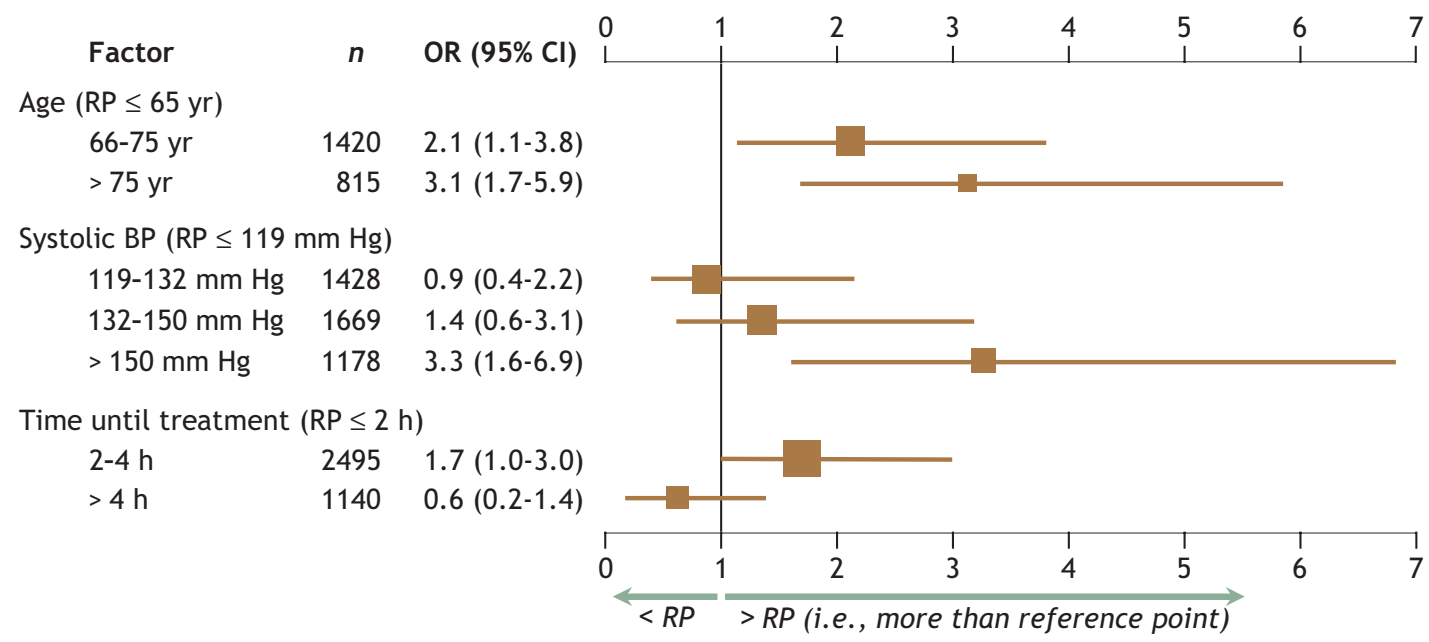

Fig. 2: The main effects, in terms of odds ratios (ORs, shown in the graph as boxes) and $95 \%$ confidence intervals $(\mathrm{Cl}$, shown as bars), of independent factors predicting the occurrence of intracranial hemorrhage relative to the reference points of age $\leq 6_{5}$ years, systolic blood pressure $\leq 119 \mathrm{~mm} \mathrm{Hg}$ and time from symptom onset to treatment $\leq 2$ hours $(N=5717)$. The relative importance of these 3 measures, as indicated by their $\chi^{2}$ values, is 15,17 and 10 , respectively. There was a 4 -way age-sex-treatment-trial interaction $(p=0.005)$. The area under the receiver-operator curve (AUC index) for this logistic regression was 0.728 .

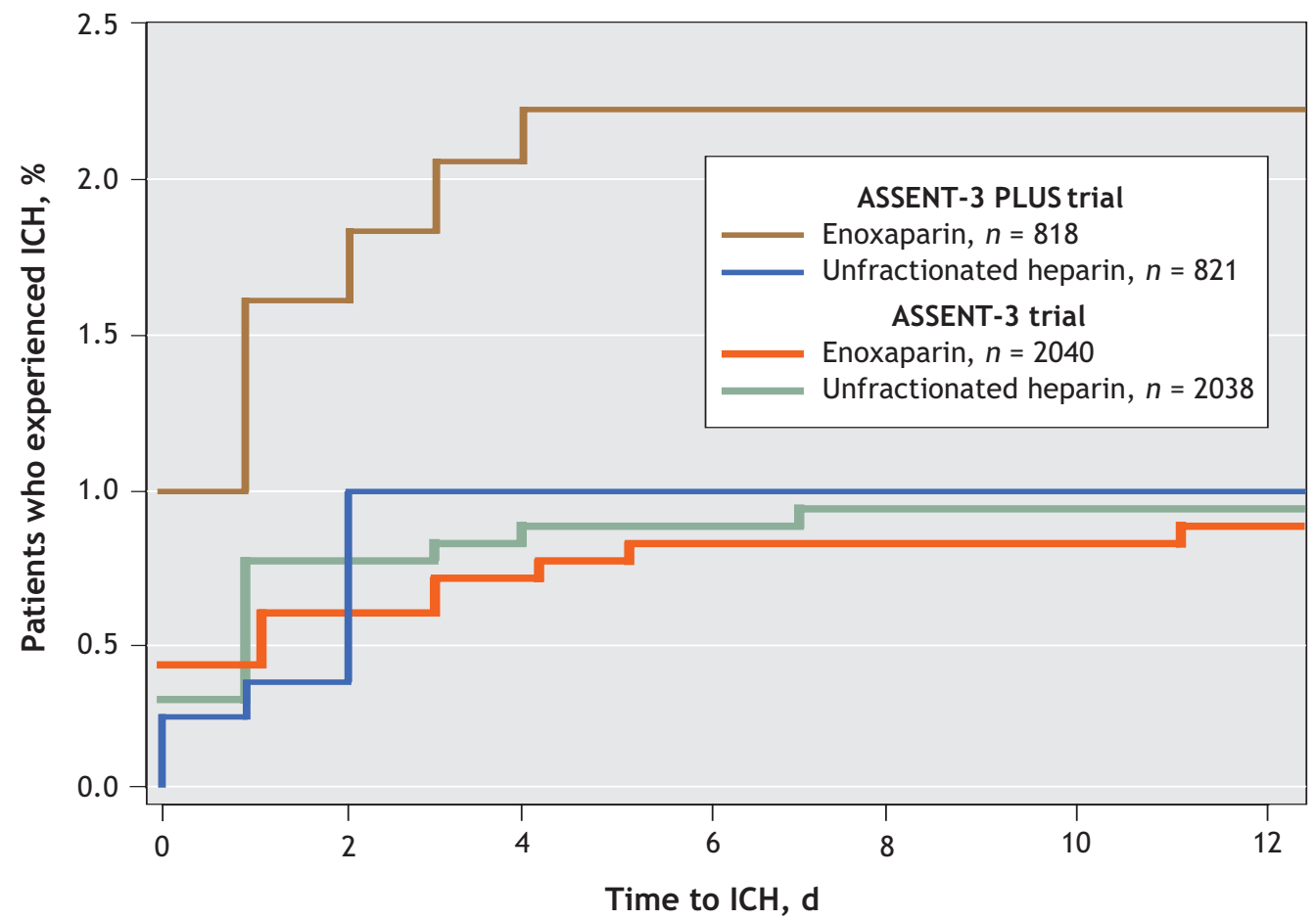

Fig. 3: Cumulative occurrence of intracranial hemorrhage $(\mathrm{ICH})$ by antithrombotic treatment group. Data from the enoxaparin group from the ASSENT-3 PLUS trial were compared with those from the ASSENT-3 trial $(p=0.01)$, and with data from the unfractionated heparin groups of the ASSENT-3 PLUS $(p=0.047)$ and ASSENT-3 $(p=0.004)$ trials, by means of the log-rank test. 
over the more aggressive antithrombotic heparin strategy in ASSENT-2. ${ }^{1,8,9}$ About 1 in 5 patients who were randomly assigned prehospital to a treatment group were not assigned a Killip class; this, along with our inability to discern which patients in ASSENT- 3 arrived by ambulance, somewhat limits our intertrial comparison and suggests a need for caution in interpreting the effects on intracranial hemorrhage in the ASSENT3 PLUS trial.

A consistent benefit in avoidance of reinfarction and refractory recurrent ischemia among patients treated with enoxaparin was evident in both trials. We report a novel insight into a partitioning of treatment effect among those 1 in 5 patients (I8\%) undergoing urgent revascularization. This amplification of treatment benefit in a high-risk group that otherwise exhibits a higher rate of recurrent $\mathrm{MI}$ is consistent with the benefit amplification that occurs when glycoprotein IIb/IIIa inhibitors are used in conjunction with revascularization in non-ST-segment elevation acute coronary syndromes. ${ }^{10}$ The intervals until myocardial reinfarction or revascularization were recorded only in days; since $65 \%$ of reinfarctions (IIo/I68) occurred on the same day as revascularization, we cannot evaluate what proportion of reinfarctions led to urgent revascularization versus those that resulted from invasive procedures. Our post hoc subgroup analyses therefore require further validation. ${ }^{11}$ Notwithstanding this benefit in patients who underwent revascularization, the increased frequency of major systemic bleeding among nonrevascularized patients over the duration of the hospital stay raises a question as to whether the duration of enoxaparin therapy is appropriately balanced with the risk-benefit ratio. Since the majority of recurrent infarctions, refractory ischemia and urgent revascularizations occur soon after presentation, it may be prudent to further explore the optimal duration of enoxaparin therapy.

In summary, our pooled analysis of ASSENT- 3 and ASSENT- 3 PLUS data showed that the combination of tenecteplase and enoxaparin was associated with a robust reduction in reinfarction and refractory ischemia, augmentation in major systemic bleeding and a propensity for increased intracranial hemorrhage among elderly patients ( $>75$ years), especially women. ${ }^{12}$ This last finding suggests not only a need for caution but also a need for further studies involving this growing segment of the population, which has high rates of adverse events after treatment of STEMI.

Since acceptance of our article for publication, findings from the ExTRACT (Enoxaparin and Thrombolysis Reperfusion for Acute Myocardial Infarction) trial, a large study of fibrinolysis after STEMI that compared enoxaparin (a reduced dose, in patients over the age of 75) with unfractionated heparin, have been published..$^{13}$ Those investigators also found a a reduction in the overall risk of death and nonfatal reinfarction with enoxaparin, associated with an increased risk of major bleeding but without any significant increase in risk of intracranial hemorrhage.

This article has been peer reviewed.

From the University of Alberta (Armstong, Chang), Edmonton, Alta.; University Hospital (Wallentin), Uppsala, Sweden; CHRU Centre Hospitalier Régional Universitaire de Lille (Goldstein), Lille, and Boehringer Ingelheim
France (Danays), Reims, France; Duke Clinical Research Institute (Granger), Durham, NC; and Biostatistical Centre (Bogaerts) and University Hospital (Werf), Gasthuisberg, Leuven, Belgium

Competing interests: None declared for Wei-Ching Chang. Paul Armstrong has received research grants and honoraria from Hoffmann-LaRoche Canada; Christopher Granger, from Sanofi-Aventis and Genentech Inc.; and both authors, from Boehringer-Ingelheim and Aventis Pharmaceuticals. Lars Wallentin, Patrick Goldstein, Kris Bogaerts and Frans Van de Werf have received research grants from Boehringer-Ingelheim, Germany. Thierry Danays is Scientific Director of Boehringer-Ingelheim, France. Frans Van de Werf serves on the speakers' bureaus of Boehringer-Ingelheim and Aventis Pharmaceuticals.

Contributors: All authors contributed to the study conception and design; data acquisition, analysis and interpretation; and the drafting and critical revision of the manuscript. All authors approved the version to be published.

Acknowledgements: We thank Cherry Robinson for her editorial assistance.

The study was supported by Boehringer Ingelheim, Germany; Genentech, South San Francisco, Calif.; and Aventis, Bridgewater, NJ.

\section{REFERENCES}

I. Assessment of the Safety and Efficacy of a New Thrombolytic Regimen (ASSENT)3 Investigators. Efficacy and safety of tenecteplase in combination with enoxaparin, abciximab, or unfractionated heparin: the ASSENT-3 randomized trial in acute myocardial infarction. Lancet 2001;358:605-13.

2. Wallentin L, Goldstein P, Armstrong PW, et al. Efficacy and safety of tenecteplase in combination with the low-molecular-weight heparin enoxaparin or unfractionated heparin in the prehospital setting: the Assessment of the Safety and Efficacy of a New Thrombolytic Regimen (ASSENT)-3 PLUS randomized trial in acute myocardial infarction. Circulation 2003;108:135-42.

3. Hosmer DW, Lemeshow S. Applied logistic regression. New York: Wiley; I989.

4. Herlitz J, Karlson BW, Bang A, et al. Characteristics and outcome for patients with acute chest pain in relation to whether or not they were transported by ambulance. Eur JEmerg Med 2000;7:195-200.

5. Canto JG, Zalenski RJ, Ornato JP, et al. for the National Registry of Myocardial Infarction 2 Investigators. Use of emergency medical services in acute myocardial infarction and subsequent quality of care. Observations from the National Registry of Myocardial Infarction 2. Circulation 2002;106:3018-23.

6. Widimsk" P, Budesinsky T, Vorac D, et al.; PRAGUE Study Group Investigators. Long distance transport for primary angioplasty vs immediate thrombolysis in acute myocardial infarction. Final results of the randomized national multicentre trial-PRAGUE-2. Eur Heart J 2003;24:94-I04.

7. Andersen HR, Nielsen T, Rasmussen K, et al. for the DANAMI-2 Investigators. A comparison of coronary angioplasty with fibrinolytic therapy in acute myocardial infarction. N Engl J Med 2003;349:733-42.

8. Ryan TJ, Antman EM, Brooks NH, et al. I9g9 Update: ACC/AHA guidelines for the management of patients with acute myocardial infarction: Executive summary and recommendations. A report of the American College of Cardiology/American Heart Association Task Force on Practice Guidelines (Committee on Management of Acute Myocardial Infarction). Circulation I999;100:I0I6-30.

9. Assessment of the Safety and Efficacy of a New Thrombolytic Regimen Investigators. Single-bolus tenecteplase compared with front-loaded alteplase in acute myocardial infarction: the ASSENT-2 double-blind randomized trial. Lancet 1999; 354:716-22.

Io. Braunwald E, Antman EM, Beasley JW, et al. ACC/AHA 2002 Guideline update for the management of patients with unstable angina and non-ST segment elevation myocardial infarction: a report of the American College of Cardiology/American Heart Association Task Force on Practice Guidelines (Committee on the Management of Patients with Unstable Angina). J Am Coll Cardiol 2002;40:1366-74.

II. Pieper KS, Tsiatis AA, Davidian M, et al. Differential treatment benefit of platelet glycoprotein IIb/IIIa inhibition with percutaneous coronary intervention versus medical therapy for acute coronary syndromes: Exploration of methods. Circulation 2004;I09:64I-6.

12. Simoons ML, Maggioni AP, Knatterud G, et al. Individual risk assessment for intracranial haemorrhage during thrombolytic therapy. Lancet 1993;342(8886-7): I523-8.

I3. Antman EM, Morrow DA, McCabe $\mathrm{CH}$, et al; for the ExTRACT-TIMI 25 Investigators. Enoxaparin versus unfractionated heparin with fibrinolysis for ST-elevation myocardial infarction. New Engl J Med 2006;354(I4):I477-88.

Correspondence to: Dr. Paul W. Armstrong, University of Alberta, 5 I Medical Sciences Building, Rm. 2, Edmonton $A B$ T6G $2 \mathrm{H}_{7}$; fax 780 492-9486; paul.armstrong@ualberta.ca 